\title{
Generalized Beatty sequences and complementary triples
}

\author{
J.-P. Allouche \\ CNRS, IMJ-PRG, Sorbonne Université \\ 4 Place Jussieu \\ F-75252 Paris Cedex 05 France \\ jean-paul.allouche@imj-prg.fr
}

\author{
F. M. Dekking \\ Delft University of Technology \\ Faculty EEMCS, P.O. Box 5031 \\ 2600 GA Delft, The Netherlands \\ F.M.Dekking@math.tudelft.nl
}

May 23, 2019

\begin{abstract}
A generalized Beatty sequence is a sequence $V$ defined by $V(n)=p\lfloor n \alpha\rfloor+q n+r$, for $n=1,2, \ldots$, where $\alpha$ is a real number, and $p, q, r$ are integers. These occur in several problems, as for instance in homomorphic embeddings of Sturmian languages in the integers. Our results are for the case that $\alpha$ is the golden mean, but we show how some results generalize to arbitrary quadratic irrationals. We mainly consider the following question: For which sixtuples of integers $p, q, r, s, t, u$ are the two sequences $V=(p\lfloor n \alpha\rfloor+q n+r)$ and $W=(s\lfloor n \alpha\rfloor+t n+u)$ complementary sequences?

We also study complementary triples, i.e., three sequences $V_{i}=\left(p_{i}\lfloor n \alpha\rfloor+q_{i} n+r_{i}\right), i=1,2,3$, with the property that the sets they determine are disjoint with union the positive integers.
\end{abstract}

Keywords: Generalized Beatty sequences, Complementary pairs and triples, morphic words, return words, Kimberling transform

MSC: 11B83, 11B85, 68R15, 11D09, 11J70

\section{Introduction}

A Beatty sequence is the sequence $A=(A(n))_{n \geq 1}$, with $A(n)=\lfloor n \alpha\rfloor$ for $n \geq 1$, where $\alpha$ is a positive real number. What Beatty observed is that when $B=(B(n))_{n \geq 1}$ is the sequence defined by $B(n)=\lfloor n \beta\rfloor$, with $\alpha$ and $\beta$ satisfying

$$
\frac{1}{\alpha}+\frac{1}{\beta}=1,
$$

then $A$ and $B$ are complementary sequences, that is, the sets $\{A(n): n \geq 1\}$ and $\{B(n): n \geq 1\}$ are disjoint and their union is the set of positive integers. In particular if $\alpha=\varphi=\frac{1+\sqrt{5}}{2}$ is the golden ratio, this gives that the sequences $(\lfloor n \varphi\rfloor)_{n \geq 1}$ and $\left(\left\lfloor n \varphi^{2}\right\rfloor\right)_{n \geq 1}$ are complementary.

Among the numerous results on Beatty sequences, a paper of Carlitz, Scoville and Hoggatt $\underline{6}$. Theorem 13, p. 20] studies the monoid generated by $A=(A(n))_{n \geq 1}$ and $B=(B(n))_{n \geq 1}$ for the composition of sequences in the case where $\alpha$ is equal to $\varphi=\frac{1+\sqrt{5}}{2}$, the golden ratio. (The composition of two integer sequences $U=(U(n))_{n \geq 1}$ and $V=(V(n))_{n \geq 1}$ is the sequence $U V:=U \circ V=(U(V(n)))_{n \geq 1}$, so that the monoid generated by $A$ and $B$ is composed of sequences like $A^{k} B^{j} A^{\ell} \ldots$, where $A^{k}=A A \ldots A$ is the composition of $k$ sequences equal to $A$.)

Theorem 1 (Carlitz-Scoville-Hoggatt) Let $U=(U(n))_{n \geq 1}$ be a composition of the sequences $A=$ $(\lfloor n \varphi\rfloor)_{n \geq 1}$ and $B=\left(\left\lfloor n \varphi^{2}\right\rfloor\right)_{n \geq 1}$, containing $i$ occurrences of $A$ and $j$ occurrences of $B$, then for all $n \geq 1$

$$
U(n)=F_{i+2 j} A(n)+F_{i+2 j-1} n-\lambda_{U},
$$

where $F_{k}$ are the Fibonacci numbers $\left(F_{0}=0, F_{1}=1, F_{n+2}=F_{n+1}+F_{n}\right)$ and $\lambda_{U}$ is a constant. 
Definition We call generalized Beatty sequence any sequence $V$ of the type $V(n)=p(\lfloor n \alpha\rfloor)+q n+r, n \geq 1$, where $\alpha$ is a real number, and $p, q$, and $r$ are integers.

Two examples of generalized Beatty sequences are $U=A A$ and $U=A B$, where Theorem 1 gives $A A(n)=A(n)+n-1$, and $A B(n)=2 A(n)+n$. These two formulas directly imply the following result.

Corollary 2 Let $V$ be a generalized Beatty sequence given by $V(n)=p(\lfloor n \varphi\rfloor)+q n+r, n \geq 1$. Then $V A$ and $V B$ are generalized Beatty sequences with parameters $\left(p_{V A}, q_{V A}, r_{V A}\right)=(p+q, p, r-p)$ and $\left(p_{W A}, q_{W A}, r_{W A}\right)=$ $(2 p+q, p+q, r)$.

As an extension of Beatty's observation the following natural questions can be asked.

Question 1 Let $\alpha$ be an irrational number, and let $A$ defined by $A(n)=\lfloor n \alpha\rfloor$ for $n \geq 1$ be the Beatty sequence of $\alpha$. Let $\operatorname{Id}$ defined by $\operatorname{Id}(n)=n$ be the identity map on the integers. For which sixtuples of integers $p, q, r, s, t, u$ are the two sequences $V=p A+q \operatorname{Id}+r$ and $\quad W=s A+t \operatorname{Id}+u$ complementary sequences?

Question 2 For which nonuples of integers $\left(p_{1}, q_{1}, r_{1}, p_{2}, q_{2}, r_{2}, p_{3}, q_{3}, r_{3}\right)$ are the three sequences $V_{i}=$ $p_{i} A+q_{i} \mathrm{Id}+r_{i}, i=1,2,3$ a complementary triple, i.e., the sets they determine are disjoint with union the positive integers 1 .

Remark 3 The theorem of Carlitz, Scoville and Hoggatt above was rediscovered by Kimberling [22, Theorem 5, p. 3]: it is thus attributed to Kimberling in, e.g., [15, p. 575], [16, p. 647], [24, p. 20-21]. This was corrected in [5, Theorem 2, p. 2]. Theorem 1 in [17] is also a special case of the theorem of Carlitz, Scoville and Hoggatt.

Remark 4 The four papers [27, [4, 20] and [18] consider different generalizations of Beatty sequences.

Remark 5 One can ask whether the monoid generated by other complementary sequences by composition can be written as a subset of the set of linear combinations of a finite number of elements. Some answers for Beatty sequences can be found in the rich paper of Fraenkel [14 (see, e.g., p. 645). Another, possibly unexpected, example is given by the Thue-Morse sequence. Namely call odious (resp. evil) the integers whose binary expansion contains an odd (resp. even) number of 1's, then it was proved in [2, Corollaries 1 and 3] that the sequences $(A(n))_{n \geq 0}$ and $(B(n))_{n \geq 0}$ of odious and evil numbers satisfy for all $n$

$$
\begin{aligned}
& A(n)=2 n+1-t(n), \quad B(n)=2 n+t(n), \quad A(n)-B(n)=1-2 t(n) \\
& A(A(n))=2 A(n), \quad B(B(n))=2 B(n), \quad A(B(n))=2 B(n)+1, \quad B(A(n))=2 A(n)+1 .
\end{aligned}
$$

where $(t(n))_{n \geq 0}$ is the Thue-Morse sequence, i.e., the characteristic function of odious integers. (This sequence can be defined by $t(0)=0$ and for all $n \geq 0, t(2 n)=t(n)$ and $t(2 n+1)=1-t(n)$.) This easily implies that any finite composition of $(A(n))_{n \geq 0}$ and $(B(n))_{n \geq 0}$ can be written as $(\alpha A(n)+\beta B(n)+\gamma)_{n \geq 0}$, since $t(A(n))=1$ and $t(B(n))=0$ for all $n$.

\section{Complementary pairs}

Let $\alpha$ be an irrational number, and let $A$ defined by $A(n)=\lfloor n \alpha\rfloor$ for $n \geq 1$ be the Beatty sequence of $\alpha$. In this section we consider Question 1 of the Introduction, which we call the Complementary pair problem.

In what follows we will require that as a function $A: \mathbb{N} \rightarrow \mathbb{N}$ is injective, since we then have a 1-to-1 correspondence between sequences and subsets of $\mathbb{N}$. (See 21] for non-injective Beatty sequences.)

In the case that $V$ and $W$ are increasing, we will also require, without loss of generality, that $V(1)=1$. Solutions $(p, q, r, s, t, u)$ with $p=0$ or $s=0$ will be called trivial.

\footnotetext{
${ }^{1}$ And when is this partition "nice"? (in Fraenkel's terminology [14, a "nice" integer DCS -Disjoint Covering System).
} 
The homogeneous Sturmian sequence generated by a real number $\alpha \in(0,1)$ is the sequence

$$
c_{\alpha}:=(\lfloor(n+1) \alpha\rfloor-\lfloor n \alpha\rfloor)_{n \geq 1} .
$$

(For more about Sturmian sequences, the reader can consult, e.g., [26, Ch. 2].)

A real number $\alpha$ is called a Sturm number if $\alpha \in(0,1)$ is a quadratic irrational number with algebraic conjugate $\bar{\alpha}$ satisfying $\bar{\alpha} \notin(0,1)$. Sturm numbers have a property that is useful to recognize their generalized Beatty sequences.

Proposition 6 ([9], [1]) Let $\alpha$ be a Sturm number. Then there exists a morphism $\sigma_{\alpha}$ on the alphabet $\{0,1\}$, such that $\sigma_{\alpha}\left(c_{\alpha}\right)=c_{\alpha}$.

In the following we will consider the variants of $\sigma_{\alpha}$ on various other alphabets than $\{0,1\}$, but will not indicate this in the notation. The following lemma is implied trivially by

$$
V=p A+q \mathrm{Id}+r \Rightarrow V(n+1)-V(n)=p(A(n+1)-A(n))+q=p c_{\alpha}(n)+q .
$$

Lemma 7 Let $\alpha$ be a Sturm number. Let $V=(V(n))_{n \geq 1}$ be the generalized Beatty sequence defined by $V(n)=p(\lfloor n \alpha\rfloor)+q n+r$, and let $\Delta V$ be the sequence of its first differences. Then $\Delta V$ is the fixed point of $\sigma_{\alpha}$ on the alphabet $\{q, p+q\}$.

We remark that it can be shown that the first letters of $\sigma_{\alpha}(0)$ and $\sigma_{\alpha}(1)$ are equal (see, e.g., 12]), so $\sigma_{\alpha}$ has a unique fixed point. It is also obvious that this fixed point starts with 0 if $\alpha \in(0,1 / 2)$, and with 1 if $\alpha \in(1 / 2,1)$. For general $\alpha$, one replaces $\alpha$ with $\breve{\alpha}=\alpha-\lfloor\alpha\rfloor(=\{\alpha\})$. When $\alpha$ is the golden mean $\varphi=(1+\sqrt{5}) / 2$, the morphism generating the sequence associated to the Sturm number $\breve{\varphi}=\varphi-1$ is $0 \mapsto 1,1 \mapsto 10$, so one has to exchange 0 and 1 if one wishes to compare $\Delta V$ with the classical Fibonacci morphism $0 \mapsto 01,1 \mapsto 0$. As a special case of Lemma 7 we therefore obtain one direction of the following lemma.

Lemma 8 Let $V=(V(n))_{n \geq 1}$ be the generalized Beatty sequence defined by $V(n)=p(\lfloor n \varphi\rfloor)+q n+r$, and let $\Delta V$ be the sequence of its first differences. Then $\Delta V$ is the Fibonacci word on the alphabet $\{2 p+q, p+q\}$. Conversely, if $x_{a b}$ is the Fibonacci word on the alphabet $\{a, b\}$, then any $V$ with $\Delta V=x_{a b}$ is a generalized Beatty sequence $V=((a-b)\lfloor n \varphi\rfloor)+(2 b-a) n+r)$ for some integer $r$.

Another observation is that the $q \mathrm{Id}+r$ part in a generalized Beatty sequence generates arithmetic sequences. The following lemma, which will be useful in proving Theorem 11, shows that in some weak sense the Wythoff part $p A$ of a $g$ eneralized Beatty sequence is orthogonal to its arithmetic sequence part, provided that $\frac{1}{3}<\{\alpha\}<\frac{2}{3}$, where $\{\alpha\}=\alpha-\lfloor\alpha\rfloor$. We prove this for $\frac{4}{3}<\alpha<\frac{5}{3}$.

Lemma 9 Let $\alpha$ satisfy $\frac{4}{3}<\alpha<\frac{5}{3}$, and let $V=(V(n))_{n \geq 1}$ be the generalized Beatty sequence defined by $V(n)=p(\lfloor n \alpha\rfloor)+q n+r$ with $p \neq 0$, then neither $(V(1), V(2), V(3))$, nor $(V(2), V(3), V(4))$ can be an arithmetic sequence of length 3.

Proof: When $\frac{3}{2}<\alpha<\frac{5}{3}$, we have $\lfloor\alpha\rfloor=1,\lfloor 2 \alpha\rfloor=3$, and $\lfloor 3 \alpha\rfloor=4,\lfloor 4 \alpha\rfloor=6$, so

$$
\begin{aligned}
& V(2)-V(1)=p\lfloor 2 \alpha\rfloor+2 q+r-p\lfloor\alpha\rfloor-q-r=2 p+q, \\
& V(3)-V(2)=p\lfloor 3 \alpha\rfloor+3 q+r-p\lfloor 2 \alpha\rfloor-2 q-r=p+q, \\
& V(4)-V(3)=p\lfloor 4 \alpha\rfloor+4 q+r-p\lfloor 3 \alpha\rfloor-3 q-r=2 p+q,
\end{aligned}
$$

and the result follows, since $p \neq 0$. When $\frac{4}{3}<\alpha<\frac{3}{2}$, we have $\lfloor\alpha\rfloor=1,\lfloor 2 \alpha\rfloor=2,\lfloor 3 \alpha\rfloor=4$, and $\lfloor 4 \alpha\rfloor=5$. So this time $V(2)-V(1)=p+q, V(3)-V(2)=2 p+q$ and $V(4)-V(3)=p+q$, leading to the same conclusion.

Remark 10 We note for further use that solving the equations in the proof of Lemma 9 for $p$ and $q$, supplemented with an equation for $r$, yields in the case $\frac{3}{2}<\alpha<\frac{5}{3}$ that

$$
\left\{\begin{array}{l}
p=-V(1)+2 V(2)-V(3) \\
q=V(1)-3 V(2)+2 V(3) \\
r=V(1)+V(2)-V(3)
\end{array}\right.
$$


Let $\alpha=\varphi$ be the golden mean. Then the classical solution is $(p, q, r)=(1,0,0)$ and $(s, t, u)=(1,1,0)$, which corresponds to the Beatty pair $(\lfloor n \varphi\rfloor),\left(\left\lfloor n \varphi^{2}\right\rfloor\right)$. Another solution is given by

$$
(p, q, r)=(-1,3,-1), \quad(s, t, u)=(1,2,0),
$$

which corresponds to the Beatty pair $(\lfloor n(5-\sqrt{5}) / 2\rfloor),(\lfloor n(5+\sqrt{5}) / 2\rfloor)$, which is equal to

$$
(\lfloor n(3-\varphi)\rfloor),(\lfloor n(\varphi+2)\rfloor) .
$$

Theorem 11 Let $\alpha=\varphi$. Then there are exactly two non-trivial increasing solutions to the complementary pair problem: $(p, q, r, s, t, u)=(1,0,0,1,1,0)$ and $(p, q, r, s, t, u)=(-1,3,-1,1,2,0)$.

Proof: Recall that $V(1)=1$. Note that $V(2)<5$, since otherwise $(W(1), W(2), W(3))=(2,3,4)$, which is not allowed by Lemma 9. There are therefore three cases to consider, according to the value of $V(2)$.

1. The case $V(1)=1, V(2)=2$. Then by Lemma 9, $V(3)=3$ is not possible.

(a) If $V(3)=4$, then, by Remark 10 $p=-1, q=3, r=-1$, which is one of the two solutions.

(b) If $V(3)=5$, then, by Remark [10, $p=-2, q=5, r=-2$, which implies that $V(4)=6, V(5)=7$, $V(6)=10$. So $W(1)=3, W(2)=4, W(3)=8$, which gives $s=-3, t=7, u=-1$ (Remark 10 applied to $W$ ), implying $W(5)=10$, which contradicts complementarity.

(c) If $V(3)=m$ with $m>5$, then $W(1)=3, W(2)=4, W(3)=5$, which contradicts Lemma 9

2. The case $V(1)=1, V(2)=3$.

(a) If $V(3)=4$, then, by Remark 10, $p=1, q=0, r=0$, which is one of the two solutions.

(b) If $V(3)=5$, then we obtain a contradiction with Lemma 9 ,

(c) If $V(3)=6$, then, by Remark 10, $p=-1, q=4, r=-2$, which implies $V(5)=10$. But we must then have $W(1)=2, W(2)=4, W(3)=5$, so (Remark 10 applied to $W), s=1, t=0, u=1$, which implies $W(6)=10$, a contradiction with complementarity.

(d) If $V(3)=m$ with $m>6$, then we obtain a contradiction with Lemma 9 since then $W(2)=4$, $W(3)=5, W(4)=6$.

3. The case $V(1)=1, V(2)=4$.

(a) If $V(3)=5$, then, by Remark 10, $p=2, q=-1, r=0$, thus $V(4)=8$; hence $W(1)=2$, $W(2)=3, W(3)=6$. Hence, by Remark 10 applied to $W, s=-2, t=5, u=-1$, so that $W(5)=8=V(4)$, which contradicts complementarity.

(b) If $V(3)=6$, then $W(1)=2, W(2)=3, W(3)=5$. Thus, by Remark 10 applied to $W, s=-1$, $t=3, u=0$. Hence $W(4)=6=V(3)$, which contradicts complementarity.

(c) If $V(3)=7$, then we obtain a contradiction with Lemma 9

(d) If $V(3)=m$ with $m>7$, then it follows that $V(3)=8$, since we have $W(1)=2, W(2)=3, W(3)=$ 5 , yielding, by Remark 10 applied to $W, W(n)=(-A(n)+3 n)=2,3,5,6,7,9,10,12,13,14, \ldots$ With $V(3)=8$, one obtains (by Remark 10) that $V(n)=-A(n)+5 n-3$, but then $V(5)=14=$ $W(10)$, i.e., $V$ and $W$ are not complementary.

For $\alpha=\sqrt{2}$ the classical solution to the complementary pair problem is $V=A, W=A+2 \mathrm{Id}$, i.e., the Beatty pair given by $V(n)=\lfloor n \sqrt{2}\rfloor$, and $W(n)=\lfloor n(2+\sqrt{2})\rfloor$. As $\frac{4}{3}<\sqrt{2}<\frac{3}{2}$, we can use Lemma 9 and adapt Remark 10 to prove the following result, in the same way as Theorem [1]

Theorem 12 Let $\alpha=\sqrt{2}$. Then there is a unique non-trivial increasing solution to the complementary pair problem: $(p, q, r, s, t, u)=(1,0,0,1,2,0)$.

We end this section with an example where $\{\alpha\} \notin\left(\frac{1}{3}, \frac{2}{3}\right)$. 
Theorem 13 Let $\alpha=\sqrt{8}$. Then there is a unique non-trivial increasing solution to the complementary pair problem: $(p, q, r, s, t, u)=(1,4,0,-1,4,0)$.

Proof: Since $(4+\sqrt{8}, 4-\sqrt{8})$ is a Beatty pair, $(p, q, r, s, t, u)=(1,4,0,-1,4,0)$ is a solution to the complementary pair problem. To prove that it is unique is more involved. We fix $V(1)=1$.

Let $\breve{\alpha}=\alpha-2=\sqrt{8}-2$. Then $\breve{\alpha} \in(0,1)$, and $\breve{\alpha}$ has the periodic continued fraction expansion $[0 ; \overline{1,4}]$. It follows then from [9], or from Corollary 9.1.6 in [3] that the morphism $\sigma_{\breve{\alpha}}$ fixing the homogeneous Sturmian sequence $c_{\breve{\alpha}}$ is given by

$$
\sigma_{\breve{\alpha}}: 0 \mapsto 11110, \quad 1 \mapsto 111101
$$

Note that

$$
V(n)=p\lfloor n \sqrt{8}\rfloor+q n+r=p\lfloor n(\sqrt{8}-2)\rfloor+(2 p+q) n+r=p\lfloor n \breve{\alpha}\rfloor+(2 p+q) n+r .
$$

The difference sequence $\Delta V$ of $V$ is therefore the fixed point of $\sigma_{\breve{\alpha}}$ on the alphabet $\{2 p+q, 3 p+q\}$. Since we require $V$ to be increasing, both $2 p+q$ and $3 p+q$ have to be larger than 0 . We split the possibilities according to the value of $3 p+q$. The arguments below are based on the fact, following from the form of $\sigma_{\breve{\alpha}}$, that $V$ starts with an arithmetic sequence of length 5 , followed by an arithmetic sequence of length 6 , both with common differences $3 p+q$, and separated by a distance $2 p+q$.

1. The case $3 p+q \geq 3$.

If $3 p+q \geq 3$, then $W(1)=2, W(2)=3$, so $W=s A+t \operatorname{Id}+u$ has to start with an arithmetic sequence of length 5 with common difference 1 , i.e., $W(1), W(2), \ldots, W(5)=2,3, \ldots, 6$. Moreover, since $W(6)=7$ is not possible (it would imply $p=0$ ), $V(2)=7$, which implies $V(3)=13$. But then the second arithmetic sequence of $W$, which has length 6 , does not fit in between $V(2)$ and $V(3)$.

2. The case $3 p+q=2$.

In this case $V(1), \ldots, V(5)=1,3,5,7,9$, so $W(1), \ldots, W(5)=2,4,6,8,10$. Then either $V(6)=11$, or $W(6)=11$.

In the former case we must have $2 p+q=V(6)-V(5)=2$, which implies $p=0$, which is trivial. In the latter case $W(6), \ldots, W(11)=11,13,15,17,19,21$, and $W(12)=22$, since $W(12)-W(11)=$ $W(6)-W(5)=1$. But also, $V(6), \ldots, V(11)$ equals $12,14, \ldots, 22$. So $V$ and $W$ are not complementary.

3. The case $3 p+q=1$.

In this case $V(1), \ldots, V(5)=1,2,3,4,5$, so $W(1)=6$, since $V(6)=6$ would imply $p=0$. Then either $V(6)=7$, or $W(2)=7$.

In the former case, $V(6), \ldots, V(11)=7,8,9,10,11,12$, and $W(2)=13$. This implies that $2=V(6)-$ $V(5)=2 p+q$, which leads to $(p, q, r)=(-1,4,0)$, and $(s, t, u)=(1,4,0)$, which is the announced solution.

In the latter case $W(1), \ldots, W(5)=6,7,8,9,10$, and $V(6)=11$. So $2 p+q=V(6)-V(5)=5$. This implies $(p, q, r)=(-5,16,-5)$, and $(s, t, u)=(-6,19,-1)$. But then $V(12)=22$, and $W(11)=22$. So $V$ and $W$ are not complementary.

\subsection{Generalized Pell equations}

If $V$ and $W$ are not increasing, then an analysis as in the proof of Theorem 11 is still possible, but very lengthy. We therefore consider another approach in this subsection. Considering the densities of $V$ and $W$ in $\mathbb{N}$, one sees that a necessary condition for $(p A+q \operatorname{Id}+r, s A+t \operatorname{Id}+u)$ to be a complementary pair is that

$$
\frac{1}{p \alpha+q}+\frac{1}{s \alpha+t}=1
$$

In what follows we concentrate on the case $\alpha=\varphi=(1+\sqrt{5}) / 2$, but our arguments can be generalized to the case of arbitrary quadratic irrationals. 
Proposition 14 A necessary condition for the pair $V=p A+q \mathrm{Id}+r$ and $W=s A+t \mathrm{Id}+u$ to be $a$ complementary pair is that $p \neq 0$ is a solution to the generalized Pell equation

$$
5 p^{2} x^{2}-4 x=y^{2}, \quad x, y \in \mathbb{Z}
$$

Proof: Using $\varphi^{2}=1+\varphi$, a straightforward manipulation shows that (2) implies

$$
(p s+p t+q s-p-s) \varphi=q+t-p s-q t .
$$

But since $\varphi$ is irrational, this can only hold if

$$
p s+p t+q s-p-s=0, \quad q+t-p s-q t=0 .
$$

The first equation gives $p t=p-(p+q-1) s$. Eliminating $p t$ from $p^{2} s+(q-1) p t-p q=0$, we obtain $p^{2} s+(p-(p+q-1) s)(q-1)-p q=0$. This gives the quadratic equation

$$
s q^{2}+(p-2) s q-\left(p^{2}+p-1\right) s+p=0 .
$$

Since $q$ is an integer, $\Delta:=(p-2)^{2} s^{2}+4 s\left(\left(p^{2}+p-1\right) s-p\right)$ has to be an integer squared. Trivial manipulations yield that

$$
\Delta=5 p^{2} s^{2}-4 p s .
$$

Since $p$ divides the square $\Delta, 5 p^{2} s^{2}-4 p s=p^{2} y^{2}$ for some integer $y$, and hence $p$ also divides $s$. If we put $s=p x$, we obtain $5 p^{3} x^{2}-4 p^{2} x=p^{2} y^{2}$, which finishes the proof of the proposition.

Actually there is a simple characterization of the integers $p$ such that the Diophantine equation above has a solution.

Proposition 15 The generalized Pell equation

$$
5 p^{2} x^{2}-4 x=y^{2}, \quad x, y \in \mathbb{Z}
$$

has a solution for $p>0$ if and only if $p$ divides some Fibonacci number of odd index, i.e., if and only $p$ divides some number in the set $\{1,2,5,13,34, \ldots\}$.

Proof: First suppose that there are integers $p>0$ and $x, y \in \mathbb{Z}$ such that $5 p^{2} x^{2}-4 x=y^{2}$. Let $d:=\operatorname{gcd}(x, y)$ and $x^{\prime}=x / d, y^{\prime}=y / d$, so that $\operatorname{gcd}\left(x^{\prime}, y^{\prime}\right)=1$. We thus have

$$
5 p^{2} d x^{\prime 2}-4 x^{\prime}=d y^{\prime 2} .
$$

Thus $x^{\prime}$ divides $d y^{\prime 2}$, but it is prime to $y^{\prime}$, hence $x^{\prime}$ divides $d$. Since clearly $d$ divides $4 x^{\prime}$, we have $d=\alpha x^{\prime}$ for some $\alpha$ dividing 4 , hence $\alpha$ belongs to $\{1,2,4\}$. This yields $\alpha\left(5 p^{2} x^{\prime 2}-y^{\prime 2}\right)=4$. We distinguish three cases.

1. If $\alpha=1$, then we have $5 p^{2} x^{2}-y^{\prime 2}=4$. But the equation $5 X^{2}-4=Y^{2}$ has an integer solution if and only if $X$ is a Fibonacci number with odd index [25. p. 91]. Hence $p x^{\prime}$ must be a Fibonacci number with odd index, thus $p$ divides a Fibonacci number with odd index.

2. If $\alpha=2$, then we have $5 p^{2} x^{\prime 2}-y^{\prime 2}=2$. Note that $x^{\prime}$ must be odd, otherwise $x^{\prime}$ and $y^{\prime}$ would be even, which contradicts $\operatorname{gcd}\left(x^{\prime}, y^{\prime}\right)=1$. Thus $5 p^{2} x^{\prime 2} \equiv p^{2} \bmod 4$, hence $p^{2}-2 \equiv y^{\prime 2} \bmod 4$. If $p$ is even, this yields $y^{\prime 2} \equiv 2 \bmod 4$, while if $p$ is odd, this gives $y^{\prime 2} \equiv 3 \bmod 4$. There is no such $y^{\prime}$ in both cases.

3. If $\alpha=4$, then we have $5 p^{2} x^{\prime 2}-y^{\prime 2}=1$, thus $5\left(2 p x^{\prime}\right)^{2}-\left(2 y^{\prime}\right)^{2}=4$, then $2 p x^{\prime}$ must be a Fibonacci number with odd index, thus $p$ divides a Fibonacci number with odd index.

Now suppose that $p$ divides some Fibonacci number with odd index, say there exists a $k$ with $F_{2 k+1}=p \beta$. We will construct an integer solutions in $(x, y)$ to the equation $5 p^{2} x^{2}-4 x=y^{2}$. We know (again [25, p. 91]) that there exists some integer $\gamma$ with $5 F_{2 k+1}^{2}-4=\gamma^{2}$ thus $5 p^{2} \beta^{2}-4=\gamma^{2}$. Let $x=\beta^{2}$ and $y=\beta \gamma$. Then

$$
5 p^{2} x^{2}-4 x=5 p^{2} \beta^{4}-4 \beta^{2}=\beta^{2}\left(5 p^{2} \beta^{2}-4\right)=\beta^{2} \gamma^{2}=y^{2} .
$$


Corollary 16 There are no solutions to the complementary pair problem if -1 is not a square modulo $p$, i.e., if $p$ does not belong to the sequence $1,2,5,10,13,17,25,26,29,34,37,41, \ldots$ (sequence A008784 in [28]). This is in particular the case if $p$ has a prime divisor congruent to 3 modulo 4.

Proof: We will prove that if there are solutions to the complementary problem for $p$, thus if $p$ divides an odd-indexed Fibonacci number (Propositions 14 and 15), then -1 is a square modulo $p$. Using again the characterization in [25, p. 91], there exist two integers $x, y$ with $5 p^{2} x^{2}-4=y^{2}$. We distinguish two cases.

1. If $p$ is odd, we have $y^{2} \equiv-4 \bmod p$ and $2^{2} \equiv 4 \bmod p$. But 2 is invertible modulo $p$, hence, by taking the quotient of the two relations, we obtain that -1 is a square modulo $p$.

2. If $p$ is even, remembering that $p x=F_{2 k+1}$ for some $k$, we claim that $p$ must be congruent to 2 modulo 4 and that $x$ must be odd. Namely the sequence of odd-indexed Fibonacci numbers, reduced modulo 4 , is easily seen to be the periodic sequence $\left(\begin{array}{lll}1 & 2 & 1\end{array}\right)^{\infty}$. Hence it never takes the value 0 modulo 4 . The equality $5 p^{2} x^{2}-4=y^{2}$ implies that $y$ must be even, thus we have $5(p / 2)^{2} x^{2}-1=(y / 2)^{2}$, say $(y / 2)^{2}=-1+z(p / 2)$. Up to replacing $(y / 2)$ with $(y+p) / 2$, we may suppose that $(y / 2)$ is even (recall that $p / 2$ is odd). Thus $z(p / 2)$ is even, hence $z$ is even, say $z=2 z^{\prime}$. This gives $(y / 2)^{2}=-1+z^{\prime} p$, thus -1 is a square modulo $p$.

Remark 17 We have just seen that if the integer $p$ divides some odd-indexed Fibonacci number then -1 is a square modulo $p$ (sequence A008784 in [28]). A natural question is then whether it is true that if -1 is a square modulo $p$, then $p$ must divide some odd-indexed Fibonacci number. The answer is negative, since on one hand $12^{2} \equiv-1 \bmod 29$, and, on the other hand, the sequence of odd-indexed Fibonacci numbers modulo 29 is the periodic sequence $(1,2,5,13,5,2,1)^{\infty}$ which is never zero.

Let us look at examples of solutions to the Diophantine equation for values of $p$ that divide some Fibonacci number with odd index. Consider, for example, the case where $p=s$. Then Equation (4) becomes $\Delta=$ $5 p^{4}-4 p^{2}$, so the Diophantine equation is

$$
5 x^{2}-4=y^{2}, \quad x, y \in \mathbb{Z} .
$$

For $p=F_{1}=1$ we obtain the two sequences $V=A+r$ and $W=A+\mathrm{Id}+u$. These are complementary only when $r=u=0$, and we obtain the classical Beatty pair $(A, A+\mathrm{Id})$.

For $p=F_{3}=2$ we obtain the two sequences $V=2 A+2 \mathrm{Id}+r$ and $W=2 A-2 \mathrm{Id}+u$. These cannot be complementary for any $r$ and $u$, since for $u=0$ we have $W(n)=2(\lfloor n \varphi\rfloor)-2 n=2(\lfloor n(\varphi-1)\rfloor$, which gives all even numbers, since $\varphi-1<1$. This an example where Equation (2) does not apply, since $W$ as a function is not injective.

For $p=F_{5}=5$ we obtain the two sequences $V=5 A+4 \mathrm{Id}+r$ and $W=5 A-7 \mathrm{Id}+u$. To make these complementary we are forced to choose $r=u=3$, and we obtain

$V=(12,26,35,49,63,72,86,95,109,123,132,146,160,169,183,192,206,220,229,243,252,266, \ldots)$,

$W=(1,4,2,5,8,6,9,7,10,13,11,14,17,15,18,16,19,22,20,23,21,24,27,25,28,31,29,32,30, \ldots)$.

Now a proof that $V$ and $W$ form a complementary pair is much harder, when we let $V$ start with $V(0)=3$, to include 3 in the union. We can perform the following trick. We split $W$ into $(W(A(n)))_{n \geq 1}$, and $(W(B(n)))_{n \geq 1}$ (cf. Corollary 2). The two sequences $W A$ and $W B$ are increasing, and we can prove that $(V(n))_{n \geq 0},(W(A(n)))_{n \geq 1}$, and $(W(B(n)))_{n \geq 1}$ form a partition of the positive integers by proving that the three-letter sequence obtained by applying the morphism $0 \mapsto 1120,1 \mapsto 11100$ to the fixed point of the morphism $g$ given by $g: 0 \mapsto 01,1 \mapsto 011$, has the property that the preimages of 0,1 and 2 are precisely these three sequences. See Theorem 25 and its proof for a similar result.

For $p=F_{2 m+1} \geq 13$ it seems that we can always choose $r$ and $u$ for in such a way that we get almost complementary sequences: namely, e.g., for $p=13$ we find $q=9$ and $t=-20$. If we take $r=$ $u=9$, then we almost get a complementary pair. One finds $V=9,31,66,88,123,158,180,215, \ldots$ and $W=2,8,1,7,13,6,12,5,11,17,10 \ldots$ So 3 and 4 are missing. We thought we could prove, perhaps using something like the Lambek-Moser Theorem [23, that for all $F_{2 m+1}>5$ the two sequences are complementary excluding finitely many values, but we were not successful. 


\section{Complementary triples}

Here we will find several complementary triples consisting of sequences $V_{i}=p_{i} A+q_{i}$ Id $+r_{i}, \quad i=1,2,3$, where $A(n)=\lfloor n \alpha\rfloor$, and $\alpha$ is a real number.

It is interesting that the case $p_{1}=p_{2}=p_{3}=1$ cannot be realized. This was proved by Uspensky in 1927 , see 13 .

The case with different $\alpha_{i}$, is analysed by Tijdeman [31] for rational $\alpha_{i}, 1=1,2,3$. Also see [32] for the inhomogeneous Beatty case $\left(V_{i}(n)\right)_{n}=\left(\left\lfloor n \alpha_{i}+\beta_{i}\right\rfloor\right)_{n}, i=1,2,3$.

There is one triple in which we will be particularly interested (see Theorem 25):

$$
\left(\left(p_{1}, q_{1}, r_{1}\right),\left(p_{2}, q_{2}, r_{2}\right),\left(p_{3}, q_{3}, r_{3}\right)\right)=((2,-1,0),(4,3,2),(2,-1,2)) .
$$

We allow that the sequences $\left(V_{i}\right)$ are each indexed either by $\{0,1,2, \ldots\}$ or by $\{1,2, \ldots\}$.

\subsection{Two classical triples}

In this subsection $\alpha$ is always the golden mean $\varphi$. Let once more $A(n)=\lfloor n \varphi\rfloor$ for $n \geq 1$ be the terms of the lower Wythoff sequence, and let $B$ be given by $B(n)=\left\lfloor n \varphi^{2}\right\rfloor$ for $n \geq 1$, the upper Wythoff sequence. Then we have the disjoint union

$$
A(\mathbb{N}) \cup B(\mathbb{N})=\mathbb{N} .
$$

Since $B=A+\mathrm{Id}$, this is the classical complementary pair $((1,0,0),(1,1,0))$.

Here is a way to create complementary triples from complementary pairs.

Proposition 18 Let $(V, W)$ be a golden mean complementary pair $V=p A+q \operatorname{Id}+r$ and $W=s A+t \operatorname{Id}+u$. Then $\left(V_{1}, V_{2}, V_{3}\right)$ is a complementary triple, where the three parameters of $V_{1}$ are $(p+q, p, r-p)$, those of $V_{2}$ are $(2 p+q, p+q, r)$, and $V_{3}=W$.

Proof: Substituting Equation (5) in $V(\mathbb{N}) \cup W(\mathbb{N})=\mathbb{N}$ we obtain the disjoint union

$$
V(A(\mathbb{N})) \cup V(B(\mathbb{N})) \cup W(\mathbb{N})=\mathbb{N} .
$$

Then Corollary 2 implies the statement of the proposition.

Remark 19 Actually Proposition 18 and Corollary 2 can be generalized to cover an infinite family of quadratic irrationals, but their statements will not be true for all quadratic irrationals. We hope to revisit this point in a future article.

Applying Proposition 18 to the basic complementary pair $((1,0,0),(1,1,0))$ gives that

$$
((1,1,-1),(2,1,0),(1,1,0)) \text { and }((1,0,0),(2,1,-1),(3,2,0))
$$

are complementary triple:2, which we will call classical triples. The first classical triple is given at the end of Skolem's 1957 paper 30].

Let $w=1231212312312 \ldots$ be the fixed point of the morphism

$$
1 \mapsto 12,2 \mapsto 3,3 \mapsto 12 .
$$

Then $w^{-1}(1)=A A, w^{-1}(2)=B$ and $w^{-1}(3)=A B$ give the three sequences $V_{1}, V_{3}$ and $V_{2}$ of the first classical triple (see [10).

The question arises: is there also a morphism generating the second triple? The answer is positive.

\footnotetext{
${ }^{2}$ In 28] these are (A003623, A003622, A001950) and (A000201, A035336, A101864).
} 
Proposition 20 Let $\left(V_{1}, V_{2}, V_{3}\right)=(A, 2 A+\mathrm{Id}-1,3 A+2 \mathrm{Id})=(A, B A, B B)$. Then $\left(V_{1}, V_{2}, V_{3}\right)$ is a complementary triple. Let $\mu$ be the morphism on $\{1,2,3\}$ given by

$$
\mu: 1 \mapsto 121,2 \mapsto 13,3 \mapsto 13,
$$

with fixed point $z$. Then $z^{-1}(1)=V_{1}, z^{-1}(2)=V_{2}$ and $z^{-1}(3)=V_{3}$.

Proof: The four words of length 3 occurring in the infinite Fibonacci word $x_{\mathrm{F}}$ are 010,100,001,101. Coding these with the alphabet $\{1,2,3,4\}$ in the given order, they generate the 3 -block morphism $\hat{f}_{3}$ that describes the successive occurrences of the words of length 3 in $x_{\mathrm{F}}$ (cf. [10]). It is given by

$$
\hat{f}_{3}(1)=12, \quad \hat{f}_{3}(2)=3, \quad \hat{f}_{3}(3)=14, \quad \hat{f}_{3}(4)=3 .
$$

It has just one fixed point, which is

$$
z^{\prime}:=1,2,3,1,4,1,2,3,1,2,3,1,4,1,2,3, \ldots
$$

We claim that

$$
z^{\prime-1}(1)=A A, z^{-1}(2)=B A, \quad z^{\prime^{-1}}(3)=A B, \quad z^{\prime-1}(4)=B B .
$$

To see this, note that the 3 -block 010 in $x_{\mathrm{F}}$ uniquely decomposes as $010=f(0) 0$. It follows that the $m^{\text {th }}$ occurrence of 010 in $x_{\mathrm{F}}$ corresponds exactly to the $m^{\text {th }}$ occurrence of 0 in $f^{-1}\left(x_{\mathrm{F}}\right)=x_{\mathrm{F}}$. This implies that the positions of the occurrences of 010 are of the form $A(A(n))$, and also that the occurrences of 001 are of the form $A(B(n))$, since $B(\mathbb{N})$ is the complement of $A(\mathbb{N})$.

For the 3-block 100, we note that it always occurs in $x_{\mathrm{F}}$ as factor of 0100 , which uniquely decomposes in $x_{\mathrm{F}}$ as $0100=f^{2}(0) 0$. It follows that the $m^{\text {th }}$ occurrence of 100 in $x_{\mathrm{F}}$ corresponds exactly to the $m^{\text {th }}$ occurrence of 0 in $f^{-2}\left(x_{\mathrm{F}}\right)=x_{\mathrm{F}}$. This implies that the positions of the occurrences of 100 are of the form $B(A(n))$, and also that the occurrences of 101 are of the form $B(B(n))$.

Since the 0's in $x_{\mathrm{F}}$ occur either as prefix of 001, or of 010, we see that we have to merge the letters 1 and 3 to obtain the sequence $A$. This is not possible with $\hat{f}_{3}$. However, the square of this 3 -block morphism is given by

$$
1 \mapsto 123,2 \mapsto 14,3 \mapsto 123,4 \mapsto 14,
$$

and now we can consistently merge 1 and 3 to the single letter 1 , obtaining the morphism $\mu$, after mapping 4 to 3 . Under this projection the sequence $z^{\prime}$ maps to $z$.

\subsection{Non-classical triples}

Let $\mathcal{L}$ be a language, i.e., a sub-semigroup of the free semigroup generated by a finite alphabet under the concatenation operation. A homomorphism of $\mathcal{L}$ into the natural numbers is a map $\mathrm{S}: \mathcal{L} \rightarrow \mathbb{N}$ satisfying $\mathrm{S}(v w)=\mathrm{S}(v)+\mathrm{S}(w)$, for all $v, w \in \mathcal{L}$.

Let $\mathcal{L}_{\mathrm{F}}$ be the Fibonacci language, i.e., the set of all words occurring in the Fibonacci word $x_{\mathrm{F}}$, the iterative fixed point of the morphism $f$ defined on $\{0,1\}^{*}$ by $f: 0 \mapsto 01,1 \mapsto 0$. The following result is proved in [11].

Theorem 21 ([1]) Let $\mathrm{S}: \mathcal{L}_{\mathrm{F}} \rightarrow \mathbb{N}$ be a homomorphism. Define $a=\mathrm{S}(0), b=\mathrm{S}(1)$. Then $\mathrm{S}\left(\mathcal{L}_{\mathrm{F}}\right)$ is the union of the two generalized Beatty sequences $((a-b)\lfloor n \varphi\rfloor+(2 b-a) n)$ and $((a-b)\lfloor n \varphi\rfloor+(2 b-a) n+a-b)$.

For a few choices of $a$ and $b$, the two sequences in $\mathrm{S}\left(\mathcal{L}_{\mathrm{F}}\right)$ and the sequence $\mathbb{N} \backslash \mathrm{S}(\mathcal{L})$ form a complementary triple of generalized Beatty sequences. The goal of this section is to prove this for $a=3, b=1$. It turns out that the three sequences

$$
(2\lfloor n \varphi\rfloor-n)_{n \geq 1},(2\lfloor n \varphi\rfloor-n+2)_{n \geq 1},(4\lfloor n \varphi\rfloor+3 n+2)_{n \geq 0},
$$

form a complementary triple.

Remark 22 Note that the indices for $(4\lfloor n \varphi\rfloor)+3 n+2)_{n \geq 0}$ are $(n \geq 0)$, not $(n \geq 1)$ 
It is easy to see that the Fibonacci word $x_{\mathrm{F}}$ can be obtained as an infinite concatenation of two kinds of blocks, namely 01 and 001 (part (i) of Lemma 23 below). Kimberling introduced in the OEIS 28] the sequence A284749 obtained by replacing in this concatenation every block 001 by 2 . We let $x_{\mathrm{K}}=A 284749$ denote this sequence.

Lemma 23 Let $f, g, h, k$ be the morphisms defined on $\{0,1\}^{*}$ by

$$
f: 0 \mapsto 01,1 \mapsto 0 ; \quad g: 0 \mapsto 01,1 \mapsto 011 ; \quad h: 0 \mapsto 01,1 \mapsto 001 ; \quad k: 0 \mapsto 01,1 \mapsto 2 .
$$

Then

$$
\text { (i) } x_{\mathrm{F}}=f^{\infty}(0)=h\left(g^{\infty}(0)\right), \quad \text { (ii) } x_{\mathrm{K}}=k\left(g^{\infty}(0)\right) \text {. }
$$

Proof:

(i) An easy induction proves that for all $n \geq 0$ one has $h g^{k}=f^{2 k} h$. (Note that it suffices to prove that the values of both sides are equal when applied to 0 and to 1.) By letting $n$ tend to infinity this implies $h g^{\infty}(0)=f^{\infty}(0)$.

(ii) Assertion (i) gives that $x_{\mathrm{F}}$ is an infinite concatenation of blocks $h(0)=01$ and $h(1)=001$, obtained as image under $h$ from $g^{\infty}(0)$. So substituting 2 for 001 in $x_{\mathrm{F}}$ is the same as substituting 01 for 0 and 2 for 1 in $g^{\infty}(0)$.

It is interesting that $x_{\mathrm{K}}$ is fixed point of a morphism $i$, given by $i: 0 \mapsto 01,1 \mapsto 2,2 \mapsto 0122$. . This follows from the relation $k g^{n}=i^{n+1}$ for all $n$, which is easily proved by induction.

Lemma 24 Define the morphism $\ell$ from $\{0,1\}^{*}$ to $\{0,1,2\}^{*}$ by $\ell: 0 \mapsto 012,1 \mapsto 0022$. Then the sequence $v=\left(v_{n}\right)_{n \geq 1}=\ell\left(g^{\infty}(0)\right)$ is obtained from $x_{\mathrm{K}}$ by replacing 1 by 0 in all blocks 0122 (but not in 0120 ).

Proof: Note that $k g: 0 \mapsto 012,1 \mapsto 0122$. Lemma 24 then follows from $x_{\mathrm{K}}=k\left(g^{\infty}(0)\right)=k g\left(g^{\infty}(0)\right)$.

Theorem 25 Let $v$ be the sequence defined above, i.e., $v=\ell\left(g^{\infty}(0)\right)$, where $g$ and $\ell$ are the morphisms defined by $g: 0 \mapsto 01,1 \mapsto 011$ and $\ell: 0 \mapsto 012,1 \mapsto 0022$. Then the increasing sequences of integers defined by $v^{-1}(0), v^{-1}(1), v^{-1}(2)$ form a partition of the set of positive integers $\mathbb{N}^{*}$. Furthermore

- $v^{-1}(0)=\{1,4,5,8,11,12,15,16,19,22, \ldots\}$ is equal to the sequence of integers $(2\lfloor n \varphi\rfloor-n)_{n \geq 1}$, where $\varphi$ is the golden ratio $\frac{1+\sqrt{5}}{2}$ (sequence A050140 in [28]),

- $v^{-1}(1)=\{2,9,20,27, \ldots\}$ is equal to the sequence of integers $(4\lfloor n \varphi\rfloor+3 n+2)_{n \geq 0}$.

- $v^{-1}(2)=\{3,6,7,10,13,14,17,18,21,24, \ldots\}$ is equal to the sequence of integers $\left((2\lfloor n \varphi\rfloor-n+2)_{n \geq 1}\right)$ (i.e., $2+\mathrm{A} 050140)$.

Proof: We see from Lemma 24 that the positions of 2 in $v$ are the same as the positions of 2 in $x_{\mathrm{K}}$. In Section 4 it is proved that $x_{\mathrm{K}}^{-1}(2)=((2\lfloor n \varphi\rfloor-n+2)$, see Example 30, and so the third assertion of the theorem follows.

Inspection of the occurrences of 0 and 2 in $\ell(0)$ and $\ell(1)$ then shows that the first assertion will also be true.

For the proof of the second assertion consider $\ell(01)=0120022, \ell(011)=01200220022$. Since $g^{\infty}(0)$ is a concatenation of the words $g(0)=01$ and $g(1)=011$, we see from this that the differences of indices of the positions where 1's in $v$ occur are 7 or 11 , and moreover, that a 0 in $g^{\infty}(0)$ generates a difference 7 , a 1 in $g^{\infty}(0)$ generates a difference 11 .

It is well known and easy to prove that $g^{\infty}(0)$ equals the binary complement of the Fibonacci word prefixed with the letter 1 . From this it follows that $\Delta v^{-1}(1)$ is the Fibonacci word on the alphabet $\{11,7\}$. Now Lemma 8 gives the generalized Beatty sequence $V=(4\lfloor n \varphi\rfloor+3 n+2)$. The first element 2 in $v^{-1}(1)$ is obtained by letting $V$ start at $n=0$ instead of $n=1$.

Remark 26 Some of the sequences above are images of Sturmian sequences by a morphism. Namely $v=\ell\left(g^{\infty}(0)\right), x_{\mathrm{K}}=k\left(g^{\infty}(0)\right)$. Such sequences are examples of sequences called quasi-Sturmian in [7]. Their block complexity is of the form $n+C$ for $n$ large enough ( $C=1$ for Sturmian sequences). These sequences were studied, e.g., in [29], 8], and [7. 


\section{Generalized Beatty sequences and return words}

In this section we show that generalized Beatty sequences are closely related to return words.

Theorem 27 Let $x_{\mathrm{F}}$ be the Fibonacci word, and let $w$ be any word in the Fibonacci language $\mathcal{L}_{\mathrm{F}}$. Let $Y$ be the sequence of positions of the occurrences of $w$ in $x_{\mathrm{F}}$. Then $Y$ is a generalized Beatty sequence, i.e., for all $n \geq 0, Y(n+1)=p\lfloor n \varphi\rfloor+q n+r$ with parameters $p, q, r$, which can be explicitly computed.

Proof: Let $x_{\mathrm{F}}=r_{0}(w) r_{1}(w) r_{2}(w) r_{3}(w) \ldots$, written as a concatenation of return words of the word $w$ (cf. [19, Lemma 1.2). According to Theorem2.11 in [19], if we skip $r_{0}(w)$, then the return words occur as the Fibonacci word on the alphabet $\left\{r_{1}(w), r_{2}(w)\right\}$. Thus the distances between occurrences of $w$ in $x_{\mathrm{F}}$ are equal to $l_{1}:=\left|r_{1}(w)\right|$ and $l_{2}:=\left|r_{2}(w)\right|$. We can apply Lemma 8, which yields $p=l_{1}-l_{2}, q=2 l_{2}-l_{1}$. Inserting $n=0$, we find that $r=\left|r_{0}(w)\right|+1$, as the first occurrence of $w$ is at the beginning of $r_{1}(w)$.

\subsection{The Kimberling transform}

Here we will obtain non-classical triples appearing in another way, namely as the three indicator functions $x^{-1}(0), x^{-1}(1)$ and $x^{-1}(2)$, of a sequence $x$ on an alphabet $\{0,1,2\}$ of three symbols. In our examples the sequence $x$ is a 'transform' $\mathcal{T}\left(x_{\mathrm{F}}\right)$ of the Fibonacci word $x_{\mathrm{F}}=01001010010010100 \ldots$ These transforms have been introduced by Kimberling in the OEIS [28]. Our main example is: $\mathcal{T}$ : [001 $\mapsto 2]$. Replacing, in $x_{\mathrm{F}}=01001010010010100 \ldots$, each 001 by 2 gives $x_{\mathrm{K}}=01201220120 \ldots$.

For the transform method $\mathcal{T}$ we can derive a 'general' result similar to Theorem 27. However, since Kimberling applies the StringReplace procedure from Mathematica, which replaces occurrences of $w$ consecutively from left to right, we do not obtain a sequence of return words in the case that $w$ has overlaps in $x_{\mathrm{F}}$. This restricts the number of words $w$ to which the following theorem (Theorem 29) applies. We first recall a definition from [34, p. 593-594].

Definition 28 (Wen \& Wen) Let $w$ be a factor of the Fibonacci word $x_{\mathrm{F}}$. We say that $w$ has an overlap in $x_{\mathrm{F}}$ if there exist non-empty words $x, y$ and $z$ such that $w=x y=y z$, and the word $x y z$ is a factor of the Fibonacci word.

Theorem 29 Let $x_{\mathrm{F}}$ be the Fibonacci word, and let $w$ be a factor of $x_{\mathrm{F}}$ that has no overlap in $x_{\mathrm{F}}$. Consider the transform $\mathcal{T}\left(x_{\mathrm{F}}\right)$, which replaces every occurrence of the word $w$ in $x_{\mathrm{F}}$ by the letter 2 . Let $Y$ be the sequence $\left(\mathcal{T}\left(x_{\mathrm{F}}\right)\right)^{-1}(2)$, i.e., the positions of 2 's in $\mathcal{T}\left(x_{\mathrm{F}}\right)$. Then $Y$ is a generalized Beatty sequence, i.e., for all $n \geq 1, Y(n)=p\lfloor n \varphi\rfloor+q n+r$, with parameters $p, q, r$, which can be explicitly computed.

Proof: As in the proof of Theorem 27 let $x_{\mathrm{F}}=r_{0}(w) r_{1}(w) r_{2}(w) \ldots$, written as a concatenation of return words of the word $w$. Now the distances between 2's in $\mathcal{T}\left(x_{\mathrm{F}}\right)$ are equal to $l_{1}:=\left|r_{1}(w)\right|-|w|+1$ and $l_{2}:=\left|r_{2}(w)\right|-|w|+1$. We can apply Lemma 8 , which gives $p=l_{1}-l_{2}, q=2 l_{2}-l_{1}$. Inserting $n=1$, we find that $r=\left|r_{0}(w)\right|-l_{2}+1$.

Example 30 We take $\mathcal{T}$ : $[001 \mapsto 2]$, with image $\mathcal{T}\left(x_{\mathrm{F}}\right)=01201220120 \ldots$, so $Y=(3,6,7,10, \ldots)$. Here $r_{0}(w)=01, r_{1}(w)=00101, r_{2}(w)=001$. This gives $l_{1}=3, l_{2}=1$, implying $p=2, q=-1$ and $r=2$. So $Y$ is the generalized Beatty sequence $\left(Y_{n}\right)_{n \geq 1}=(2\lfloor n \varphi\rfloor-n+2)_{n \geq 1}$.

The question arises whether not only $\mathcal{T}\left(x_{\mathrm{F}}\right)^{-1}(2)$, but also $\mathcal{T}\left(x_{\mathrm{F}}\right)^{-1}(0)$ and $\mathcal{T}\left(x_{\mathrm{F}}\right)^{-1}(1)$ are generalized Beatty sequences. In general this is not true. However, this holds for $\mathcal{T}:[001 \mapsto 2]$.

Theorem 31 Let $\mathcal{T}:[001 \mapsto 2]$, and let $x_{\mathrm{K}}:=\mathcal{T}\left(x_{\mathrm{F}}\right)$. Then the three sequences $x_{\mathrm{K}}^{-1}(0), x_{\mathrm{K}}^{-1}(1), x_{\mathrm{K}}^{-1}(2)$ form a complementary triple of generalized Beatty sequences. 
Proof: According to Example 30 we have that $x_{\mathrm{K}}^{-1}(2)=(2\lfloor n \varphi\rfloor-n+2)_{n \geq 1}$. Since clearly $x_{\mathrm{K}}^{-1}(0)=x_{\mathrm{K}}^{-1}(1)-1$, our remaining task is to prove that $(Z(n))_{n \geq 0}:=x_{\mathrm{K}}^{-1}(1)$ is a generalized Beatty sequence. The return word structure of the word $w=001$ in $x_{\mathrm{F}}$ is given by

$$
r_{0}(w)=01, \quad r_{1}(w)=00101, \quad r_{2}(w)=001
$$

Note that $Z(0)=2$, the 1 coming from $r_{0}(w)$. This is exactly the reason why it is convenient to start $Z$ from index 0: the other 1's are coming from the $r_{1}(w)$ 's-note that $r_{2}(w)$ is mapped to 2.

The differences between the indices of occurrences of 2 in $x_{\mathrm{K}}$ are given by the Fibonacci word $3133131331 \ldots$, which codes the appearance of the words $r_{1}(w)$ and $r_{2}(w)$. Therefore, to obtain the differences between the indices of occurrences of 1 in $x_{\mathrm{K}}$, we have to map the word $w^{\prime}=13$ to 4 , obtaining the word $u=343443 \ldots$ To obtain a description of $u$, we apply Theorem 27 a second time with $w^{\prime}=13$. We have $r_{0}\left(w^{\prime}\right)=3$, $r_{1}\left(w^{\prime}\right)=133, r_{2}\left(w^{\prime}\right)=13$. So $l_{1}=\left|r_{1}\left(w^{\prime}\right)\right|-\left|w^{\prime}\right|+1=2$, and $l_{2}=\left|r_{2}\left(w^{\prime}\right)\right|-\left|w^{\prime}\right|+1=1$, which give $p=l_{1}-l_{2}=1, q=2 l_{2}-l_{1}=0$. The conclusion is that positions of 4 in $u$ are given by the generalized Beatty sequence $(\lfloor n \varphi\rfloor+1)_{n \geq 1}$. This forces that $u$ is nothing else than the Fibonacci word on $\{4,3\}$, preceded by 3. But then $Z$ is a generalized Beatty sequence with parameters $p=1, q=2$. Since $Z(1)=5$, we must have $r=2$, which happens to fit perfectly with the value $Z(0)=2$.

Here is an example where $\mathcal{T}\left(x_{\mathrm{F}}\right)^{-1}(0)$ and $\mathcal{T}\left(x_{\mathrm{F}}\right)^{-1}(1)$ are not generalized Beatty sequences.

Example 32 We take $\mathcal{T}:[00100 \mapsto 2]$, with image $\mathcal{T}\left(x_{\mathrm{F}}\right)=010010121010010121012 \ldots$, so $Y=(8,17,21 \ldots)$. Here $r_{0}(w)=0100101, r_{1}(w)=0010010100101, r_{2}(w)=00100101$. This gives $l_{1}=9, l_{2}=4$, so $p=5$ and $q=-1$ and $r=4$. So $Y$ is the generalized Beatty sequence $\left(Y_{n}\right)_{n \geq 1}=(5\lfloor n \varphi\rfloor-n+4)_{n \geq 1}$. The positions of 0 are given by $\left(\mathcal{T}\left(x_{\mathrm{F}}\right)\right)^{-1}(0)=1,3,4,6,10,12,13, \ldots$, with difference sequence $2,1,2,4,2,1, \ldots$, so by Lemma 8 this sequence is not a generalized Beatty sequence. However, it can be shown that $\left(\mathcal{T}\left(x_{\mathrm{F}}\right)\right)^{-1}(0)$ is a union of 4 generalized Beatty sequences, and the same holds for $\left(\mathcal{T}\left(x_{\mathrm{F}}\right)\right)^{-1}(1)$.

Here is the general result.

Theorem 33 For a non-overlapping word $w$ from the Fibonacci language let $\mathcal{T}:[w \mapsto 2]$, and let $Y:=$ $\mathcal{T}\left(x_{\mathrm{F}}\right)$. Suppose $w$ satisfies

$$
\left|r_{0}(w)\right| \leq\left|r_{1}(w)\right|-|w| \quad(\mathrm{SR} 0)
$$

Then the three sequences $Y^{-1}(0), Y^{-1}(1), Y^{-1}(2)$ are finite unions of generalized Beatty sequences.

Note that we already know by Theorem 29 that $Y^{-1}(2)$ is a single generalized Beatty sequence. The condition (SR0) states that the length of $r_{0}(w)$ is small with respect to the length of $r_{1}(w)$.

For the proof of Theorem 33 one needs the following proposition.

Proposition 34 Let $w$ be a word from the Fibonacci language, and let $r_{0}(w) r_{1}(w) r_{2}(w)$... be the return sequence of $w$ in the Fibonacci word $x_{\mathrm{F}}$. Then (i) $r_{0}(w)$ is a suffix of $r_{1}(w)$, and (ii) if $r_{2}(w)=w t_{2}(w)$, then $t_{2}(w)$ is a suffix of $r_{1}(w)$.

Proof: Let $s_{0}=1, s_{1}=00, s_{2}=101, s_{3}=00100, \ldots$ be the singular words introduced in [34]. According to [19, Theorem 1.9.] there is a unique largest singular word $s_{k}$ occurring in $w$, so we can write $w=$ $\mu_{1} s_{k} \mu_{2}$, for two words $\mu_{1}, \mu_{2}$ from the Fibonacci language. It is known - see [34] and the remarks after [19, Proposition 1.6.] — that the two return words of the singular word $s_{k}$ are

$$
r_{1}\left(s_{k}\right)=s_{k} s_{k+1}, \quad r_{2}\left(s_{k}\right)=s_{k} s_{k-1}
$$

According to [19, Lemma 3.1], the two return words of $w$ are given by

$$
r_{1}(w)=\mu_{1} r_{1}\left(s_{k}\right) \mu_{1}^{-1}, \quad r_{2}(w)=\mu_{1} r_{2}\left(s_{k}\right) \mu_{1}^{-1} .
$$

Substituting the first equation in the second, we obtain the key equation

$$
r_{1}(w)=\mu_{1} s_{k} s_{k+1} \mu_{1}^{-1}, \quad r_{2}(w)=\mu_{1} s_{k} s_{k-1} \mu_{1}^{-1}
$$


Proof of (i): We compare the return word decompositions of $x_{\mathrm{F}}$ by $s_{k}$ and by $w$ :

$$
r_{0}\left(s_{k}\right) r_{1}\left(s_{k}\right) r_{2}\left(s_{k}\right) r_{1}\left(s_{k}\right) \cdots=r_{0}(w) r_{1}(w) r_{2}(w) r_{1}(w) \cdots=r_{0}(w) \mu_{1} r_{1}\left(s_{k}\right) \mu_{1}^{-1} \mu_{1} r_{2}\left(s_{k}\right) \mu_{1}^{-1} \mu_{1} r_{1}\left(s_{k}\right) \mu_{1}^{-1} \cdots
$$

It follows that we must have $r_{0}\left(s_{k}\right)=r_{0}(w) \mu_{1}$, and so $r_{0}(w)=r_{0}\left(s_{k}\right) \mu_{1}^{-1}$. By [19, Lemma 2.3], $r_{0}\left(s_{k}\right)$ equals $s_{k+1}$, with the first letter deleted. Thus we obtain from Equation (6) that $r_{0}(w)$ is a suffix of $r_{1}(w)$.

Proof of (ii): Since $s_{k+1}=s_{k-1} s_{k-3} s_{k-1}$, by [34, Property 2], we can do the following computation, starting from Equation (6):

$$
r_{1}(w)=\mu_{1} s_{k} s_{k+1} \mu_{1}^{-1}=w \mu_{2}^{-1} s_{k+1} \mu_{1}^{-1}=w \mu_{2}^{-1} s_{k-1} s_{k-3} s_{k-1} \mu_{1}^{-1}=w \mu_{2}^{-1} s_{k-1} s_{k-3} \mu_{2} \mu_{2}^{-1} s_{k-1} \mu_{1}^{-1} .
$$

For $r_{2}(w)$ we have

$$
r_{2}(w)=\mu_{1} s_{k} s_{k-1} \mu_{1}^{-1}=w \mu_{2}^{-1} s_{k-1} \mu_{1}^{-1} .
$$

Now note that in this concatenation $\mu_{2}^{-1}$ cancels against a suffix of $w$. We claim that it also cancels against a prefix of $s_{k-1}$. This follows, since by [19, Proposition 2.5] any occurrence of $s_{k}$ in $x_{\mathrm{F}}$ is directly followed by a $s_{k+1}=s_{k-1} s_{k-3} s_{k-1}$ with the last letter deleted. It now follows that $t_{2}(w)=\mu_{2}^{-1} s_{k-1} \mu_{1}^{-1}$, and we see that this word is a suffix of $r_{1}(w)$.

Proof of Theorem 33:

From Property (ii) in Proposition 34 we obtain that the return words of $w$ can be written as

$$
r_{1}(w)=w m_{1}(w) t_{2}(w), \quad r_{2}(w)=w t_{2}(w),
$$

for some words $m_{1}(w)$ and $t_{2}(w)$. Let $Z:=Y^{-1}(2)$ be the the positions of the letter 2 . If $t_{2}(w)$ is non-empty, then any letter in $t_{2}(w)$ occurs in $Y:=\mathcal{T}\left(x_{\mathrm{F}}\right)$ in positions which are just a shift $-\left|t_{2}(w)\right|, \ldots,-1$ of $Z$, so each letter occurs according to a generalized Beatty sequence. The word $m_{1}(w)$ is never empty, and any letter in $m_{1}(w)$ occurs in $Y=\mathcal{T}\left(x_{\mathrm{F}}\right)$ in positions which are a shift of a subsequence of $Z$ (except, possibly, for the first occurrence, which then is in $\left.r_{0}(w)\right)$. This subsequence is obtained by replacing the distances $\ell_{1}$ and $\ell_{2}$ of $\Delta Z$ by $\ell_{1}+\ell_{2}$ and $\ell_{1}$. Moreover, these distances occur as the Fibonacci word $x_{\mathrm{F}}$ on the alphabet $\left\{\ell_{1}+\ell_{2}, \ell_{1}\right\}$, because $x_{\mathrm{F}}$ is invariant under $0 \mapsto 01,1 \mapsto 0$. Thus each letter in $m_{1}(w)$ occurs according to a generalized Beatty sequence. All these $\left|t_{2}(w)\right|+\left|m_{1}(w)\right|$ sequences start at index 1 . If we let the last $\left|r_{0}(w)\right|$ of these sequences start at index 0 , then we have taken into account all elements of $Y$. This works, because of Property (i) in Proposition 34 .

Here is an example where the (SR0) condition is not satisfied.

Example 35 We take $\mathcal{T}:[10100 \mapsto 2]$, with image $\mathcal{T}\left(x_{\mathrm{F}}\right)=01002100221002 \ldots$, so $Y=(5,9,10 \ldots)$. Here $r_{0}(w)=0100, r_{1}(w)=10100100, r_{2}(w)=10100$. The positions of 0 are given by the sequence $\left(\mathcal{T}\left(x_{\mathrm{F}}\right)\right)^{-1}(0)=1,3,4,7,8 \ldots$, which can be written as a union of two generalized Beatty sequences, except that the position 1 from the first 0 in $\mathcal{T}\left(x_{\mathrm{F}}\right)$ will not be in this union.

With Equation (6) we can deduce an equivalent simple formulation of condition (SR0). If $w=\mu_{1} s_{k} \mu_{2}$, then $r_{0}(w)$ equals $s_{k+1} \mu_{1}^{-1}$ with the first letter removed, and $r_{1}(w)=\mu_{1} s_{k} s_{k+1} \mu_{1}^{-1}$, so

$$
|w|=\left|\mu_{1}\right|+F_{k}+\left|\mu_{2}\right|, \quad\left|r_{0}(w)\right|=F_{k+1}-\left|\mu_{1}\right|-1, \quad\left|r_{1}(w)\right|=F_{k+1}+F_{k} .
$$

Filling this into condition (SR0) we obtain

$$
\left|\mu_{2}\right| \leq 1 \quad\left(\mathrm{SR}^{\prime}\right)
$$

Using $\left(\mathrm{SR} 0^{\prime}\right)$, together with Theorem 6 in [34, one can show that Theorem 33 does apply to at most 3 words $w$ of length $m$, for all $m \geq 2$ (in fact, only 2 , if $m$ is not a Fibonacci number).

Acknowledgments We thank the referee for useful comments. 


\section{References}

[1] C. Allauzen, Une caractérisation simple des nombres de Sturm, J. Théor. Nombres Bordeaux 10 (1998), $237-241$.

[2] J.-P. Allouche, B. Cloitre, V. Shevelev, Beyond odious and evil, Aequationes Math. 90 (2016), 341-353.

[3] J.-P. Allouche and J. Shallit, Automatic Sequences, Theory, Applications, Generalizations, Cambridge University Press, Cambridge, 2003.

[4] S. Artstein-Avidan, A.S. Fraenkel and V. Sos, A two-parameter family of an extension of Beatty sequences, Discrete Math. 308 (2008), 4578-4588.

[5] C. Ballot, On functions expressible as words on a pair of Beatty sequences, J. Integer Seq. 20 (2017), Art. 17.4.2.

[6] L. Carlitz, R. Scoville, V. E. Hoggatt, Jr., Fibonacci representations, Fibonacci Quart. 10 (1972), 1-28. [Also see L. Carlitz, R. Scoville, V. E. Hoggatt, Jr., Addendum to the paper: "Fibonacci representations", Fibonacci Quart. 10 (1972), 527-530.]

[7] J. Cassaigne, Sequences with grouped factors, in DLT'97, Developments in Language Theory III, Thessaloniki, Aristotle University of Thessaloniki, 1998, pp. 211-222.

[8] E. M. Coven, Sequences with minimal block growth, II, Math. Syst. Theory 8 (1975), 376-382.

[9] D. Crisp, W. Moran, A. Pollington and P. Shiue, Substitution invariant cutting sequences, J. Théor. Nombres Bordeaux 5, (1993), 123-137.

[10] F. M. Dekking, Morphisms, symbolic sequences, and their standard forms, J. Integer Seq. 19 (2016), Article 16.1.1.

[11] M. Dekking, The Frobenius problem for homomorphic embeddings of languages into the integers, Theoret. Comput. Sci. 732 (2018), 73-79.

[12] M. Dekking, Substitution invariant Sturmian words and binary trees, Integers 18A (2018), \#A7, 1-14.

[13] A. S. Fraenkel, Complementary systems of integers, Amer. Math. Monthly 84 (1977), 114-115.

[14] A. S. Fraenkel, Iterated floor function, algebraic numbers, discrete chaos, Beatty subsequences, semigroups, Trans. Amer. Math. Soc. 341 (1994), 639-664.

[15] A. S. Fraenkel, Complementary iterated floor words and the Flora game, SIAM J. Discrete Math. 24 (2010), 570-588.

[16] A. S. Fraenkel, From enmity to amity, Amer. Math. Monthly 117 (2010), 646-648.

[17] M. Griffiths, On a matrix arising from a family of iterated self-compositions, J. Integer Seq. 18 (2015), \#15.11.8.

[18] A.J.Hildebrand, J-X. Li, X-M. Li, Y. Xie, Almost Beatty partitions, Preprint (2018), http://arxiv.org/abs/1809.08690

[19] Y. Huang, Z.-Y. Wen, The sequence of return words of the Fibonacci sequence, Theoret. Comput. Sci. 593 (2015), 106-116.

[20] C. Kimberling, Beatty sequences and Wythoff sequences, generalized, Fibonacci Quart. 49 (2011), 195200.

[21] C. Kimberling, K. B. Stolarsky, Slow Beatty sequences, devious convergence, and partitional divergence, Amer. Math. Monthly 123 (2016), 267-273. 
[22] C. Kimberling, Complementary equations and Wythoff sequences, J. Integer Seq. 11 (2008), Art. 08.3.3.

[23] J. Lambek, L. Moser, Inverse and complementary sequences of natural numbers, Amer. Math. Monthly 61 (1954), 454-458.

[24] U. Larsson, N. A. McKay, R. J. Nowakowski, A. A. Siegel, Finding golden nuggets by reduction, Preprint (2015), https://arxiv.org/abs/1510.07155

[25] D. A. Lind, The quadratic field $Q(\sqrt{5})$ and a certain Diophantine equation, Fibonacci Quart. 6 (1968), $86-93$.

[26] M. Lothaire, Algebraic Combinatorics on Words, Cambridge University Press, 2002.

[27] A. McD. Mercer, Generalized Beatty sequences, Int. J. Math. Math. Sci., 1 (1978), 525-528.

[28] On-Line Encyclopedia of Integer Sequences, founded by N. J. A. Sloane, electronically available at http://oeis.org

[29] M. E. Paul, Minimal symbolic flows having minimal block growth, Math. Systems Theory 8 (1975), 309-315.

[30] Th. Skolem, On certain distributions of integers in pairs with given differences, Math. Scand. 5 (1957), $57-68$.

[31] R. Tijdeman, On complementary triples of Sturmian bisequences, Indag. Math. 7 (1996), 419-424.

[32] R. Tijdeman, Exact covers of balanced sequences and Fraenkel's conjecture, in Algebraic number theory and Diophantine analysis (Graz, 1998), de Gruyter, Berlin, 2000, pp. 467-483.

[33] J. V. Uspensky, On a problem arising out of the theory of a certain game, Amer. Math. Monthly 34 (1927), 516-521.

[34] Z.-X. Wen, Z.-Y. Wen, Some properties of the singular words of the Fibonacci word, European J. Combin. 15 (1994), 587-598. 